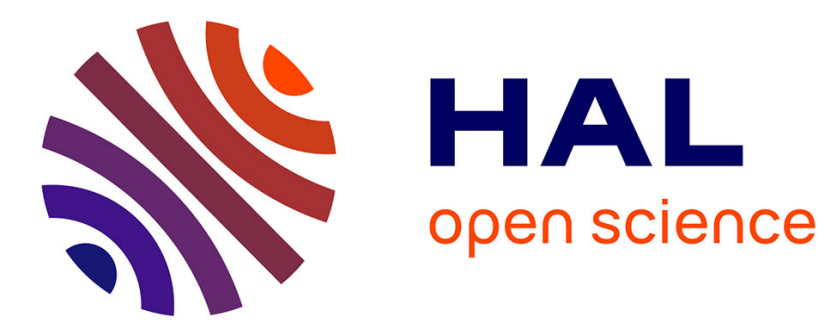

\title{
Les réseaux d'infrastructures urbaines au miroir de l'histoire: acquis et perspectives
}

\author{
Denis Bocquet
}

\section{To cite this version:}

Denis Bocquet. Les réseaux d'infrastructures urbaines au miroir de l'histoire: acquis et perspectives.

Flux - Cahiers scientifiques internationaux Réseaux et territoires, 2006, 65, pp.6-16. halshs-00128239

\section{HAL Id: halshs-00128239 \\ https://shs.hal.science/halshs-00128239}

Submitted on 1 Feb 2007

HAL is a multi-disciplinary open access archive for the deposit and dissemination of scientific research documents, whether they are published or not. The documents may come from teaching and research institutions in France or abroad, or from public or private research centers.
L'archive ouverte pluridisciplinaire HAL, est destinée au dépôt et à la diffusion de documents scientifiques de niveau recherche, publiés ou non, émanant des établissements d'enseignement et de recherche français ou étrangers, des laboratoires publics ou privés. 


\title{
Les réseaux d'infrastructures urbaines au miroir de I'histoire: acquis et perspectives
}

\author{
Denis Bocquet
}

$\mathrm{P}$ lusieurs décennies après le Networks of Power, de Thomas Parke Hughes (Hughes, 1983), la lecture de la mise en place et de l'évolution des réseaux d'infrastructures urbaines, et plus généralement l'analyse de ces phénomènes selon les méthodes $d^{\prime}$ une histoire nourrie de sciences sociales, demeure singulièrement en retard. Pourtant les réseaux techniques urbains constituent, depuis le début des années 1970, un objet d'histoire à part entière, dont l'étude a bénéficié des apports successifs de plusieurs générations de chercheurs. Tout laissait même à penser que c'était par l'histoire que les réseaux d'infrastructures, si propices supports, allaient progressivement être ouverts aux méthodes et aux questionnements des sciences sociales. L'objet de cette contribution est de tenter d'exposer les moments fondateurs de l'approche historique en matière de réseaux urbains d'infrastructures, d'en souligner les bases méthodologiques et théoriques, et, après avoir mis en évidence les blocages parfois induits par une ouverture insuffisante aux sciences sociales, de repérer ce qui, dans les tendances actuelles de la recherche, est susceptible de redonner à l'approche historique toute sa place dans les débats qui animent le monde académique.

\section{LE MOMENT T. HuGHES}

Au sein de la littérature se rapportant à l'étude de I'histoire des réseaux urbains d'infrastructures, plusieurs veines peuvent être identifiées, ayant chacune porté une part de l'évolution de la manière de considérer l'objet et des inflexions successives du lien avec d'autres manières d'étudier l'histoire, la ville, ou les sociétés en général. Mais à chaque fois, les appartenances disciplinaires et intellectuelles d'origine des chercheurs concernés ont, tout en même temps qu'elles permettaient l'innovation problématique, dessiné les limites de l'ouverture et souvent eu raison des ambitieuses velléités de construction de systèmes complexes de lecture du lien entre réseau technique urbain et société.

Le premier courant d'importance dans la maturation du paradigme historique dans l'étude des réseaux urbains d'infra- structures peut être rattaché à I'histoire de la technologie. Des auteurs comme Thomas Parke Hugues ou Wiebe Bijker en ont abondamment illustré la pertinence. À partir d'une recherche sur le degré d'influence de l'innovation technologique sur les évolutions sociales en Europe et en Amérique, ces auteurs ont peu à peu bâti une vision théorisée du rapport de la société à la technologie et à l'innovation. Mais dans cette démarche, les réseaux techniques n'étaient pas forcément au cœur de la démonstration, ni le fait urbain. Les réseaux, et la ville, n'étaient évoqués qu'en tant qu'exemples d'une évolution de la relation des sociétés humaines à la technologie. Les études dites STS (Science and Technology Studies), en somme se sont construites dans un contexte qui parfois était urbain, mais n'ont pas toujours rendu à la ville toute l'épaisseur problématique qu'elles lui prenaient.

Les premières publications de Thomas Parke Hughes étaient en effet clairement centrées sur un objet technologique (Hughes, 1964). C'est au cours des années 1980 qu'il se consacre de manière plus frontale à l'étude des réseaux en contexte urbain. En résulte le célèbre Networks of Power, consacré à l'électrification de la société occidentale, et particulièrement à l'exemple de quelques grandes villes. Là encore, le cœur de la démarche concerne l'impact d'une nouvelle technologie sur la société, sur les modes de vie, d'habiter et de consommer. Mais la vision d'un réseau en tant que tel transparaît également, de même, comme le suggère un titre resté célèbre, un rapport étroit entre réseaux techniques et réseaux de pouvoir. C'est à partir de cette expérience que Hughes s'engage dans la voie de la théorisation au sujet du rôle des infrastructures en réseau (Hugues et Mayntz, 1989) (1). Cette expérience constitue aussi le fondement d'un intérêt pour la ville, qui se concrétise par une attention prolongée à deux figures importantes, Walther Rathenau et Lewis Mumford (Hughes 1990 et Hughes \& Hughes 1990) (2). Mais la ville et les réseaux ne sont toujours pas au centre de la production de Hughes, et les livres de la fin de sa carrière, s'ils constituent des repères 
importants dans les études d'histoire de la technologie, n'ont plus particulièrement ni la ville ni les réseaux pour objet (Hughes, 2004). Pourtant, Networks of Power demeure une des références essentielles dans l'étude historicisée du rapport du réseau d'infrastructure à la ville. Mais chez Hughes, l'explication ultime, plus que dans une analyse en termes de processus sociaux, institutionnels ou territoriaux, réside souvent dans le parcours personnel de grands inventeurs ou de grands entrepreneurs. Sa vision est d'abord celle du miracle du passage de l'invention confinée à un étroit laboratoire à une application qui bouleverse les modes de vie et, surtout peut-être, le développement du capitalisme. Hughes est fasciné par les transformations du système économique sous l'impulsion d'innovations techniques, et sa lecture des réseaux, et de leur évolution, dépend fortement de ces présupposés. Sa vision de la ville en découle également. C'est déjà ainsi dans ce croisement entre destin personnel et innovation technologique que résidait le cœur de Networks of Power. À partir d'une lecture de la mise en place des réseaux de distribution d'électricité dans trois grandes villes entre la fin du XIXe siècle et le début du XXe siècle, il y construisait une typologie du lien entre réseaux et politique, destinée à marquer pour longtemps les horizons historiographiques d'une veine alors en cours de définition. On avait donc dans Networks of Power trois types de rapports entre réseaux et politique, correspondant à trois exemples emblématiques, trois villes destinées à devenir les référents plus ou moins explicites de nombreuses études ultérieures: Berlin, Chicago et Londres. Le premier type, construit à partir du cas de Berlin, était celui d'une coordination entre mise en place du réseau et politique. Le cadre de l'étude était le Berlin des années postérieures à 1870, capitale dynamique et en pleine industrialisation de la jeune Allemagne unifiée. Hughes développe à ce sujet la vision irénique d'une régulation harmonieuse par la municipalité du secteur de l'électricité, exalte la fonction régulatrice du gouvernement urbain, et surtout construit méthodiquement un lien étroit entre le parcours de deux industriels, Werner von Siemens et Emil Rathenau, et la montée en puissance du réseau d'électricité. Mais c'est au travers de la narration des phases successives du développement de l'entreprise que se construit le récit. C'est en suivant la formidable croissance d'AEG et de sa filiale BEW, dédiée à la distribution d'électricité dans la capitale, que l'on apprend les modalités de régulation telles que vues par Hughes. Celles-ci sont particulièrement analysées à partir des termes des contrats de concession négociés avec la municipalité. La fonction régulatrice du gou- vernement urbain est validée par la capacité de la mairie, d'abord en 1884, puis en 1899, à obtenir un intéressement substantiel au chiffre d'affaire et aux bénéfices de la société concessionnaire. En suivant Hughes, on arrive dans l'harmonie au tournant de 1915, qui voit la prise de contrôle sans conflit par la municipalité d'un réseau dont AEG est prête à se dégager pour augmenter ailleurs sa capacité d'investissement. Mais de très nombreuses questions demeurent en suspend, qui pourtant auraient permis de saisir plus précisément les modalités du lien entre monde de l'entreprise et institutions locales. Hughes ne donne par exemple que très peu d'indications sur la vie politique municipale, pourtant cruciales pour saisir les enjeux de la régulation. La municipalité est vue comme un ensemble peu distinct, et n'est pas l'objet du même investissement de recherche que l'entreprise. Pourtant, au-delà du constat général relatif au fonctionnement du système urbain, importe beaucoup la compréhension des processus décisionnels, des modalités de négociation ou de résolution des conflits. C'est un horizon que Hughes a peu investi. Dans le cas du Berlin du tournant du siècle, au moment de la montée du socialisme municipal, cette dimension aurait sans doute mérité une vision plus articulée, et surtout aurait mérité d'être insérée en tant qu'objet complexe dans le système explicatif global. Hughes ne s'est de même pas précisément intéressé à la dimension spatiale du réseau, et aux multiples enjeux relatifs à la constitution complexe des territoires urbains que l'attention à cette dimension aurait permis d'entrevoir. Dans le cas de Berlin surtout, où la redéfinition des limites municipales entre 1860 et 1920 est l'objet d'un constant débat, cette dimension aurait permis de tempérer la vision d'harmonie (Bernet, 2004; Bullock, 1999). Ce qui est vrai dans le cadre du Berlin de 1860 ne l'est pas forcément dans les parties de la ville qui ne seront annexées que plus tard. La ville de Hughes, en somme, est largement la projection peu spatialisée et peu politisée du rapport entre capitalisme et technologie.

Quant aux deux autres éléments de la typologie proposée par Hughes dans Networks of Power, s'ils apportent grandement à la cohérence de l'ensemble, ils contribuent également à mettre en relief les limites dans la méthode que le cas de Berlin mettait en évidence. Au sujet de Chicago par exemple, cas d'école qui sert de support au type de suprématie de la technologie sur la politique, la fascination de Hughes pour le parcours de Samuel Insull, ancien secrétaire particulier d'Edison, ancien directeur de la General Electric au moment de la fusion entre la Edison G.E. et la Thomson Houston et fondateur d'une compa- 
gnie de distribution à Chicago destinée à un brillant succès, en vient parfois à induire une vision simplifiée de la vie politique locale. Face à des hommes politiques réputés corruptibles, seule la ligne de développement de l'entrepreneur peut amener dans l'analyse la stabilité explicative nécessaire. Ce qui compte chez Hughes, c'est d'abord le parcours de l'entrepreneur. Le reste est contexte, et détermine le type, sans toutefois être l'objet d'un fort investissement de recherche. Or la vie politique locale à Chicago, entre d'une part construction d'une entité municipale dans une ville en plein boom et qui a hérité du vétuste cadre institutionnel de la Charte de l'Illinois de 1875, et d'autre part une actualité sans cesse renouvelée de scandales et de corruption, constitue bien un des éléments clés du système de relation entre réseaux et société, et mérite à ce titre d'être analysée avec les méthodes de l'histoire et des sciences sociales. Mécanismes de la décision, conflits, vecteurs de médiation, luttes d'influences, pots de vin, sont bien des éléments constitutifs d'un mode de gouvernance urbaine que I'analyse du développement des réseaux d'infrastructures permet justement de saisir. Là encore, Hughes, s'il livre à la réflexion les éléments d'une typologie destinée à marquer pour plusieurs décennies l'approche historique du rapport entre ville et réseaux d'infrastructures, n'intervient pas dans sa recherche dans toutes les aires problématiques que suggérait le cadre choisi. Sur le cas de Londres, exemple illustrant dans Networks of Power le type de la suprématie du politique sur la technique, la démarche de Hughes est comparable. Centrée sur la recherche constamment déçue d'un grand entrepreneur susceptible de faire le lien entre innovation, entreprise et ville, sa démarche arrive au constat de l'échec de personnages comme Ferranti ou Merz, signe de la suprématie des instances politiques sur la bonne volonté des entrepreneurs. Mais là encore la vie politique locale, et les détours de la construction institutionnelle et territoriale de Londres comme municipalité, ne sont pas analysés en détail. La vie politique est réduite à une explication externe, alors qu'elle constitue à l'évidence un élément essentiel du système. Le réseau lui-même est délaissé, dès lors qu'il ne correspond plus dans son développement à l'idéal recherché, non pas en terme d'efficacité, de couverture ou de justice sociale et spatiale, mais plutôt de réalisation personnelle d'un grand personnage. Hughes, en somme, qui venait d'un courant d'étude de l'histoire culturelle de la technologie, au sens d'histoire de l'impact de la technologie sur les modes de vie et sur le système économique, et dont le parcours personnel montrait un grand intérêt pour les personnages d'inventeurs et d'entrepreneurs, à la racine même de l'esprit américain, proposait une première typologie du lien entre réseaux $d^{\prime}$ 'infrastructures et société urbaine qui avait l'immense mérite de poser certaines des questions essentielles, mais butait sur les limites de la démarche même, notamment dans la confrontation avec les problématiques de la gouvernance urbaine. Or pour les sciences sociales, prime la compréhension du fonctionnement de la société. Si les réseaux constituent un bon support, et si les études fondatrices de Hughes traçaient la voie et servaient d'invitation, restait à définir plus précisément le paradigme adéquat, au croisement entre l'intérêt pour le réseau et celui pour les mécanismes sociaux qui présidaient à la décision à son égard.

Les travaux de Wiebe Bijker s'inscrivent dans une veine parallèle, et ont souvent croisé ceux de Hughes (Bijker et al., 1987). Là encore, si le cadre théorique est extrêmement utile pour penser le rapport entre société et technologie, ni les réseaux ni la ville ne constituent le point focal de la démarche (Bijker 1995, Bijker et Law 1992). La veine d'histoire culturelle de la technologie, à laquelle appartient clairement Bijker, et par laquelle il a donné une contribution fondamentale aux études STS, si elle a ouvert pour les recherches sur les réseaux les horizons prometteurs d'une analyse du lien avec la société, et a également ouvert de nouveaux liens bibliographiques, n'a pas répondu à tous les espoirs en ce qui concerne des sociétés urbaines, qui, il est vrai, ne résidaient pas forcément au cœur des problématiques des auteurs qui s'y sont reconnus. Les plus grands auteurs du courant d'histoire sociale et culturelle de la technologie, d'ailleurs, ont poursuivi leur carrière dans d'autres directions, et la production d'un chercheur comme David Nye, qui a contribué à rapprocher la réflexion sur les réseaux en tant que systèmes techniques de celles sur l'histoire de la place des mutations technologiques dans la société américaine confirme largement cette tendance (Nye, 1990, 1994, 2003) (3). De la sorte, les études sur les réseaux ont certes peu à peu intégré la réflexion sur les mutations technologiques, mais n'ont pas forcément intégré l'ensemble des problématiques propres aux sciences sociales qui auraient été susceptibles de les amener à la phase suivante de leur développement, autour par exemple d'un croisement de la thématique technologique avec celle relative au territoire dans toute sa complexité ou aux institutions, dont l'histoire se faisait de plus en plus articulée. En marge des études culturelles sur la technologie s'est ainsi développée une historiographie des réseaux qui peu à peu a eu tendance à fonctionner en vase clos. 


\section{UNE VEINE SOCIO-TECHNICIENNE}

Car depuis les années 1970 s'était largement développé un riche courant d'analyse de la place croissante des réseaux techniques dans les sociétés urbaines. Cette veine, que l'on peut qualifier, par son attention aux infrastructures urbaines, de socio-technicienne, a été largement portée par des personnages comme Joel Tarr et Gabriel Dupuy. Après un début de parcours scientifique consacré à une lecture politique des mutations urbaines à Chicago, qui constitue paradoxalement une sorte de complément avant la lettre des études de Hughes (Tarr, 1971), Joel Tarr, en collaboration avec Gabriel Dupuy, se consacre à divers travaux, aboutissant, dans les années 1980, à la publication d'une première grande somme sur la question des infrastructures urbaines en réseau (Tarr et Dupuy, 1988) (4). Mais en France, alors qu'outre Atlantique J. Tarr développe d'autres centres d'intérêt, c'est avec Georges Knaebel que Gabriel Dupuy poursuit l'exploration de cette thématique, du côté de I'histoire des techniques et d'une histoire des espaces, plutôt que d'une histoire politique, institutionnelle ou sociale (Dupuy et Knaebel, 1982). Au cours de la décennie suivante, la confrontation de cet auteur avec les approches théoriques permet la production d'importants outils de travail (1). Mais là encore, les réseaux demeurent des objets techniques dont l'étude n'inclut pas prioritairement les considérations liées au système social qui les produit. L'attention se porte surtout sur la notion de territoire et c'est de cette exploration que naissent les essais les plus stimulants. Le choix de la réflexion sur la notion de territoire prime sur l'exploration des pistes relatives à la compréhension des phénomènes sociaux et de gouvernance.

Les années 1980 voient aussi la préparation d'un important travail collectif sur Paris: Paris et ses réseaux (Caron et al., 1990). Le principal apport, outre l'importante somme d'information, réside peut-être pour cet ouvrage dans l'attention aux enjeux économiques liés aux réseaux (concession, investissement, mais aussi consommation (6)), ainsi qu'aux modes de vie qu'ils déterminent. C'est aux confins de l'histoire économique, du côté de la consommation, que l'histoire urbaine des réseaux se fait vraiment sociale. Mais là encore, la tentative n'a guère été suivie d'explorations plus systématiques. La veine économique elle-même a également été largement parcourue, notamment en France, avec une prédilection pour l'histoire des entreprises et pour la discussion de l'existence d'un éventuel modèle français de gestion des réseaux urbains d'infrastructures (Lorrain, 2002) (7). Avec les travaux de Dominique Lorrain, les réseaux sont enfin l'objet d'une lecture problématisée en fonction des méthodes des sciences sociales (Lorrain, 2005). Mais c'est essentiellement du côté de l'entreprise que se développe cette tendance et la ville et le territoire ne sont pas au cœur de la démarche. Si cela permet de participer aux débats sur les mutations de la gouvernance d'entreprise, le lien avec une vision complexe du système institutionnel et les questions spatiales demeure délicat.

Parmi les systèmes techniques urbains en réseau, les égouts, à partir des années 1980, ont par ailleurs bénéficié d'une forte attention des chercheurs. Depuis les travaux de Gérard Jacquemet sur le tout-à-l'égout, jusqu'aux études de Fabrice Laroulandie, le cas de Paris a été largement parcouru (Jacquemet, 1979, Laroulandie, 1993) (8). Mais c'est peut-être à partir du cas le Lyon, avec les travaux de Frank Scherrer, que les égouts ont été érigés en objet d'étude à part entière (Scherrer 1992) (9). La connaissance des mécanismes décisionnels ayant présidé à la mise en place des réseaux a abondamment bénéficié de ces études, qui pourtant n'ont pas suscité tout l'intérêt méthodologique qu'elles méritaient. Elles demeurent le plus souvent citées essentiellement pour leur contenu informatif. La veine $d^{\prime}$ histoire socio-technicienne des réseaux $d^{\prime}$ 'infrastructure n'a, en somme, que rarement conflué avec les grandes tendances d'une histoire urbaine elle-même en cours de consolidation. La lecture de la mise en place des infrastructures en réseau était pourtant l'occasion de croiser les perspectives, entre analyse spatiale et problématiques institutionnelle, économique, sociale, voire anthropologique et culturelle.

\section{Histoire des RÉSEAUX ET Histoire ENVIRONNEMENTALE}

Une autre inflexion importante dans la manière d'aborder l'histoire des réseaux dans sa dimension urbaine a trait aux aspects environnementaux. Mais là encore, le changement de paradigme a paradoxalement éloigné les études sur les réseaux des sciences sociales. Joel Tarr lui-même, à la fin de sa carrière, a intégré directement la dimension environnementale dans ses problématiques (Tarr, 1996). Mais c'est surtout à Martin Melosi que l'on doit la prise en compte de la dimension écologique dans l'étude des réseaux, et la confrontation avec les grandes tendances de l'histoire environnementale, d'abord avec The Sanitary City, puis avec Effluent America (Melosi, 2000) (10). Ce dernier livre contient un chapitre intitulé "Sanitary services and decision-making in Houston », promesse d'un croisement de 
I'intérêt pour les thématiques socio-environnementales et de I'analyse des processus décisionnels. Mais I'histoire environnementale, au-delà des passionnantes suggestions de Melosi, a rapidement abandonné cette piste, pour se réfugier dans une conception d'elle-même qui relève plus des sciences naturelles que des sciences sociales. Seuls peut-être les cas italien et allemand, avec par exemple le parcours scientifique de Christoph Bernhardt, Ercole Sori, Simone Neri Serneri, Elisabetta Tonizzi ou Gabriella Corona, illustrent dans l'étude des réseaux la volonté de persévérer dans le croisement progressif parmi les arguments discutés des thèmes environnementaux et des problématiques soit urbaines soit territoriales et institutionnelles (11). La récente somme dirigée par Bill Luckin, Geneviève Massard-Guibaud et Dieter Schott va également dans ce sens (Luckin et al., 2005). Mais la collaboration entre spécialistes des villes, des réseaux et de l'environnement est toujours difficile dans chacun de ces respectifs territoires académiques.

En France, c'est aussi par une attention aux sols et sous-sols urbains que les réseaux ont été abordés. Cela permettait de combiner approches juridiques, foncières, anthropologiques et techniques. L'approche a ainsi été diversifiée précocement, autour notamment des travaux de Sabine Barles et d'André Guillerme (12). Cet auteur a, de cette manière, promu une vision de l'évolution du rapport de la société urbaine à l'eau qui tienne compte de la dimension anthropologique (Guillerme, 1983) (13). Là où l'Europe continentale a pallié le relatif désintérêt anglo-saxon, malgré les impulsions de Tarr et Melosi, pour une histoire environnementale urbaine qui soit aussi science sociale, la notion a bénéficié d'une construction à même de permettre la confrontation avec d'autres tendances de la recherche, notamment dans le domaine du développement urbain durable. Mais I'histoire, là encore, se doit de trouver sa place, dans le croisement des perspectives et dans l'intégration de problématiques qui dépassent le simple horizon de ses habituels terrains d'investigation.

\section{LES RÉSEAUX TECHNIQUES URBAINS COMME SUPPORTS D'UNE HISTOIRE DES INSTITUTIONS}

L'historiographie européenne sur les réseaux techniques doit par ailleurs beaucoup à la veine des études municipales. En Italie, c'est la question de la municipalisation des services publics, au tournant du XXe siècle, qui a nourri tout d'abord I'attention à ce thème, autour par exemple de Donatella Calabi (Calabi, 1980) (14). En Allemagne, c'est de même le courant d'étude du socialisme municipal qui a le plus donné à l'histoire urbaine des réseaux d'infrastructures (Kühl, 2001). En France, à la suite des travaux de Bernard Barraqué, qui s'est aussi intéressé à la gouvernance institutionnelle des ressources, un courant parallèle s'est développé. C'est ensuite à la question de la circulation des savoirs municipaux que se sont intéressés les chercheurs, autour en France de Pierre-Yves Saunier, au Canada de Michèle Dagenais et en Italie d'Oscar Gaspari (Gaspari, 2002 ; Dogliani et Gaspari 2003, Dagenais et al., 2003), dans le contexte à la fois d'une réflexion sur les réformes urbaines et sur I'existence d'éventuels modèles et leurs modalités de transmission (15). Tout un courant de l'historiographie municipale s'est aussi intéressé à la croissance des appareils administratifs et bureaucratiques, souvent en lien avec les services techniques et la gestion des réseaux d'infrastructures. L'exemple italien, là encore, a suscité d'importants développements, avec tant les travaux de Guido Zucconi sur la mise en place, dans le contexte de I'hygiénisme, d'une bureaucratie urbaine, que ceux d'Angelo Varni et Guido Melis sur la bureaucratie (Zucconi, 1999; Varni et Melis, 1999). De nombreuses études ont en outre été consacrées dans ce pays à l'aspect institutionnel de la mise en place des appareils municipaux, et à une lecture politique et sociologique du phénomène de gonflement progressif des appareils bureaucratiques urbains. Dans le sillage de Raffaele Romanelli, des auteurs comme Aurelio Alaimo ou Marco Soresina se sont ainsi intéressés à l'évolution de la question entre la fin du XIXe siècle et le fascisme, faisant de I'Italie le pays le plus en pointe dans ce domaine (Romanelli 1989 et 1995; Alaimo 1994; Soresina 1998). Les réseaux techniques, s'ils n'étaient pas au cœur de la démarche, étaient souvent étudiés, en ce qu'ils étaient porteurs d'une bureaucratie technique et vecteurs de son développement. Mais il reste beaucoup à faire dans ce domaine, et surtout à appliquer la méthode hors de la péninsule. Pour le cas anglais, les travaux de Gloria Clifton sur le Metropolitan Board of Works londonien peuvent être rapprochés de cette production (Clifton, 1992) (16). Les études sur la bourgeoisie ont aussi contribué à l'insertion des infrastructures dans les problématiques urbaines, notamment par rapport à l'investissement des notables dans la décision publique et la vie civique (Meriggi et Schiera, 1993; Signorelli, 1999). Il en va de même pour les études sur le milieu professionnel des ingénieurs. Ayant largement porté la charge de moderniser les infrastructures urbaines à partir du XIXe siècle, cette profession s'est beaucoup investie dans les réseaux. Les études consacrées tant à la formation des ingénieurs qu'à leurs pratiques profession- 
nelles ou à leur place dans la société et la vie politique sont à ce titre précieuses pour la compréhension des enjeux liés au développement des infrastructures urbaines en réseau (Harter, 2001; Chatzis, 2000; Blanco 2000; Malatesta 1995; Picon, 1992). Les analyses relatives au courant hygiéniste le sont de même, en ce qu'elles permettent d'appréhender le lien entre une idéologie, une manière de gouverner, et des objets techniques. Un certain nombre d'études locales a également permis de progresser à la fois dans la compréhension des phénomènes de prise de décision au sujet des réseaux que dans l'intégration des thématiques politiques dans l'étude des objets techniques.

Mais d'une manière générale, ces tentatives demeurent isolées dans le milieu académique historien, et trouvent peu d'écho dans le monde de l'analyse des enjeux actuels relatifs aux réseaux et à leur gouvernance. Pourtant, là encore, I'histoire en tant que méthode pourrait apporter beaucoup, et porter avec elle les renouvellements suggérés par l'évolution des sciences sociales.

\section{LA PLACE DE L'HISTOIRE DANS LES DÉBATS CONTEMPORAINS}

Une première grande difficulté pour l'histoire des réseaux d'infrastructure dans son ouverture aux autres sciences sociales a longtemps été de dépasser les simples effets de l'assonance dans la confrontation aux études sur les réseaux de pouvoir, et, en général, sur la sociologie des organisations. La relation entre réseau et pouvoir, ou réseau et appareillage bureaucratique et institutionnel est certes présente depuis longtemps dans les études d'histoire urbaine ou d'histoire des réseaux. Ce point est même investi d'une attention particulière, à partir de plusieurs angles de vue, dont l'importance des réseaux humains (Barjot et Kurgan, 2004) (17) et la prégnance des enjeux politiques (Lorrain, 1997) (18). Mais les études sur les réseaux techniques sont longtemps restées fermées aux thématiques issues de la sphère des études sur les organisations. Or le réseau technique demeure un support précieux pour de telles études. De même, il convient sans doute de complexifier l'approche la plus courante relative aux mécanismes de la décision par le recours aux paradigmes forgés dans d'autres domaines des sciences sociales. L'historiographie italienne a tenté de rapprocher l'empirisme ambiant des études urbaines de la théorisation en cours autour de la lecture des processus décisionnels (Bobbio et Zeppetella, 1999). II reste à appliquer le programme aux réseaux d'infrastructures, formidables supports, ne serait-ce que grâce à la production d'archives que leur développement et leur gestion suscitent ou à leur ancrage territorial et temporel. L'historiographie britannique se confronte de même volontiers à la théorie, autour d'une discussion du rôle des infrastructures dans les césures sociales et territoriales. Mais il reste assurément beaucoup à faire en ce qui concerne les réseaux techniques, qui pourtant se prêtent tout à fait à une lecture détaillée de la prise de décision, de ses phases et des facteurs qui l'animent. En ce qu'il permet une sorte de micro-histoire de la décision, le réseau et les questions relatives à sa gouvernance constituent assurément un support essentiel pour le développement de I'histoire elle-même en tant que pratique de recherche. Le concept de médiation a de même permis d'importantes avancées dans l'analyse des enjeux de la décision dans la vie politique (Gherardi, 1993). Mais il y a tout à gagner à tenter de l'appliquer aussi à une lecture historique de la modernisation technique des villes à l'échelle des réseaux. En corollaire, les concepts forgés pour l'étude de la vie politique dans le domaine de la corruption sont également très utiles dans le suivi de la construction des réseaux (Melis, 1999) (19). Le réseau non seulement peut s'avérer être un bon support au déploiement de telles analyses, mais il peut également en retour contribuer au raffinement des propositions méthodologiques et théoriques.

Divers aspects semblent particulièrement importants, du poids de la politique aux enjeux complexes d'une modernisation technique des villes qui mobilise non seulement de forts intérêts économiques, mais également toute une machinerie institutionnelle et bureaucratique dont le fonctionnement et le développement sont eux-mêmes déterminés par la tâche nouvelle de construire et gérer les infrastructures. Se pose aussi, au moment de la modernisation, d'une façon inédite la question du pouvoir local, avec en discussion le rôle des municipalités, entre chambre de médiation entre pouvoir central et notabilité propriétaire locale et espace d'expression de formes nouvelles d'une démocratie urbaine. C'est une véritable construction politique de la vie urbaine que le réseau permet de saisir (Ethington, 1994). Le poids des intérêts privés ressort de même d'une manière particulièrement marquée. Mais ce qui semble émerger des études sur les réseaux urbains d'infrastructures, c'est peut-être surtout un parcours de la décision plus sinueux qu'il n'aurait pu y paraître à première vue. D'un côté les intervenants dans les processus décisionnels sont plus nombreux, et d'un autre, les motivations sont plus complexes. Apparaît également une remise en question d'un éventuel mythe du primat de la technique: plus la technique avance, plus aussi on inven- 
te dans les chemins de la médiation institutionnelle, politique ou sociale, des détours destinés à la soumettre à d'autres impératifs. En ce sens, le réseau technique est tout autant soumis au politique qu'il permet de le mettre au jour. Mais à la différence de T. Hughes, qui une fois fait le constat de la suprématie de la politique arrêtait sa démarche, déçu en quelque sorte, il convient aujourd'hui d'aller plus loin dans la démarche d'histoire, et tenter de mettre en œuvre les questionnements susceptibles d'éclairer le fonctionnement de la société, des institutions, des bureaucraties. Dans ce domaine, un certain nombre de recherches d'histoire commence de dessiner le panorama $d^{\prime}$ un renouvellement en cours. Ce sont aussi les conceptions de la modernité que le réseau permet de relativiser. L'analyse par Alice Ingold par exemple des rouages plus complexes qu'on ne l'a longtemps cru, de la transition à Milan, entre une gestion de type d'ancien régime et la modernité administrative au sujet du réseau de canaux urbains, participe de ce renouvellement (Ingold, 2003). Par la prise en compte de l'inertie des pratiques anciennes et des réseaux qui les fondent, il est possible de relire la modernité sous un jour complexifié. Pour Jérusalem de même, les recherches de Vincent Lemire montrent combien dans la gouvernance des réseaux importe ce qu'il décrit comme une concurrence des réseaux de mémoire (Lemire, 2006). L'extension des réseaux d'infrastructures dans les quartiers aux marges de la ville, souvent construits en dehors des normes de régulation de l'urbanisme, mérite également d'être soumise à des questionnements complexifiés. Pour le cas de Madrid, Charlotte Vorms a pu montrer combien l'analyse de l'équipement de ces quartiers au moment de la parenthèse républicaine révèle les césures du monde politique local et les mécanismes fins du clientélisme politique (Vorms, 2005). C'est un écho aux recherches sur la valence des normes dans les sociétés urbaines (Bocquet et De Pieri, 2005). Dans ce domaine les réseaux et leur histoire sont un support des plus précieux. Les études municipales elles-mêmes méritent, à la lumière de l'histoire des réseaux, de bénéficier des apports de méthode susceptibles de permettre l'engagement d'un dialogue interdisciplinaire: mécanismes de la vie politique, de la notabilité, de la collusion, mais aussi parcours de l'idée de bien public et de service public, tels sont les thèmes de possibles convergences. L'histoire de la constitution d'une véritable économie politique des réseaux, à la lumière de ce qu'a pu proposer Robert Millward pour la Grande-Bretagne, doit ainsi être faite, et mise à jour en fonction des thématiques les plus actuelles (Millward, 2000).
À partir du tournant du vingt et unième siècle par ailleurs, de nouveaux débats se sont ouverts autour de l'étude des réseaux techniques et de leur rapport à la ville. Un des axes principaux en a été le livre de Stephen Graham et Simon Marvin: Splintering urbanism (Graham et Marvin, 2001) (20). En attribuant aux réseaux d'infrastructure un rôle dans les effets de fragmentation urbaine, ces auteurs déplaçaient sensiblement l'attention vers le terrain d'une vision problématisée du rôle des réseaux, mais donnaient aussi à la recherche de nouvelles directions dans l'interprétation du lien entre le réseau, l'espace de la ville et le fonctionnement de la société urbaine. Si la thèse du rôle des réseaux dans une éventuelle fragmentation urbaine est largement discutée et nuancée, il demeure que la proposition a forcé chacun à questionner plus avant les liens de causalité entre système technique et territoire dans les transformations urbaines, dans la lignée de réflexions développées par exemple par J.-M. Offner et D. Pumain (Offner et Pumain, 1996) (21). C'est dans ce contexte que l'histoire peut gagner de nouveau la place qui lui revient: elle permet d'élargir le cadre de l'analyse, de mobiliser d'autres ressorts dans la démonstration, et surtout de relativiser le poids des constats faits dans l'immédiateté. Dans les débats par exemple sur l'éventuel rôle d'entraînement des premium networks, qui concentrent l'investissement au profit d'un espace restreint et de bénéficiaires choisis, seul le diagnostic historique peut permettre de dépasser les impasses du débat idéologique présent.

Il en va de même dans les recherches actuelles portant sur la gouvernance des systèmes techniques et sur les aspects liés au développement durable (Summerton, 1994, Coutard, 1999; Coutard et al., 2005). Sur tous ces points, l'histoire a beaucoup à apporter, autant dans la méthode que dans les contenus. II ne s'agit point seulement de profondeur historique, ce recul qui autorise la nuance dans les jugements, mais aussi de contribution méthodologique, dans le rapport aux sources de la connaissance et de l'information, dans le moyen de les traiter, dans les questionnements entre monde matériel et société, entre espace et société.

L'histoire a de même sa place dans les débats sur le développement urbain durable, et, d'une manière générale, sur I'analyse du lien entre société, territoire et nature. Dans la lignée des travaux d'Erik Swyngedow, qui eux-mêmes font largement appel à la dimension historique, une possible communication entre l'histoire environnementale urbaine et les protagonistes des débats actuels constitue un véritable un horizon 
d'attente dont les ressorts résident précisément dans I'application de questionnements inspirés par l'histoire institutionnelle, politique, sociale ou comportementale (Heynen et al., 2006; Baeten et Swyngedow, 2001). D’une manière générale, c'est par une conception de l'histoire qui dépasse la seule logique du recours à une information prise dans le passé et mise au service d'une démonstration, et privilégie le croisement des méthodes et des questionnements que tant les débats contemporains sur les infrastructures et l'urbain que l'histoire ellemême peuvent se nourrir mutuellement. La vision du devenir des villes globales, ainsi, a tout à gagner d'une intégration de ce type de logique de recherche. Idée de ce qu'est la ville, échelle de la décision, rapport au sol et à la nature, rôle et fonctionnement des institutions, nombreux sont les thèmes qui méritent une confrontation avec I'histoire en tant que démarche de recherche (Kaika et Swyngedow, 2000).

\section{BibLIOGRAPHIE}

AlAIMO A., 1994, L'organizzazione della città. Amministrazione e politica urbana a Bologna dopo I'Unità. 1859-1899, Bologne, II Mulino.

ANTONelLi C., 1992, " Acque sporche. Londra e il Metropolitan Board of Works (1855-1865) », Storia urbana, 61, pp. 6181.

Armstrong C. et Nelles H. V., 1986, Monopoly's Moment, Philadelphie, Temple.

Ausubel J. et Herman R. (sous la direction de), 1988, Cities and their Vital Systems, Washington, National Academy Press.

Baeten G. et Swrngedow E., 2001, "Scaling the City: The Political Economy of 'Glocal' Development », European Planning Studies, 9, n7, pp. 828-849.

Barjot D. et Kurgan G., 2004, "Les réseaux humains dans l'industrie électrique », Annales historiques de l'électricité, 2, pp. 69-87.

Barjot D., Petitet S., Varaschin D., 2002, " La concession comme levier de développement?», Entreprises et Histoire, n'31, p. 5-13.

BARLES S., 1999, La ville délétère, Seyssel, Champ Vallon.

Barles S., 2005, L'invention des déchets urbains, Seyssel, Champ Vallon.

Barles S., Breysse D., Guillerme A., Leyval C. (sous la direction de), 1999, Le sol urbain, Paris, Anthropos.

Barles S. et Guillerme A., 1995, L'urbanisme souterrain, Paris, PUF.

BARRAQUÉ B., 1984, Les services municipaux d'Annecy: espace politique local et praticiens de l'aménagement, Paris, MIR.

BARRAQué B. (sous la direction de), 1993, La ville et le génie de I'environnement, Paris, Presses de l'ENPC.

Bernhardt Ch. (sous la direction de), 2001, Environmental problems in European cities in the 19th and 20th century (Umweltprobleme in europäischen Städten des 19. und 20. Jahrhunderts), Münster, Waxmann.
Beltran A., 2004, "Quelle approche culturelle de l'histoire de l'électricité? ", Annales historiques de l'électricité, 2, pp. 139-145.

BerNet C., 2004, "The Hobrecht Plan (1862) and Berlin's urban structure », Urban History, 31-3, pp. 400-419.

Bevilaqua P. et Corona G. (sous la direction de), 2002, Ambiente e risorse nel Mezzogiorno contemporaneo, Rome, Donzelli.

BIJKER W., 1995, Of bicycles, bakelites and bulbs: toward a theory of sociotechnical change, Cambridge (Ma), MIT.

BIJKer W., Hughes T. P. et PINCH T. (sous la direction de), 1987, The Social Construction of Technological Systems. New directions in the Sociology and History of Technology, Cambridge (Ma.), MIT.

BIJKER W. et LAW J. (sous la direction de), 1992, Shaping technology/building society: studies in sociotechnical change, Cambridge (Ma.), MIT.

BlANCO L. (sous la direction de), 2000, Amministrazione, formazione e professione: gli ingegneri in Italia tra Sette e Ottocento, Bologne, II Mulino.

Bobbio L. et Zeppetella A. (sous la direction de), 1999, Perché proprio qui? Grandi opere e opposizioni locali, Milan, F. Angeli.

Bocquet D. et De Pieri F. (sous la direction de), 2005, La regola e la trasgressione, numéro spécial de la revue Storia urbana, 108.

Bocquet D. et FetTah S. (sous la direction de), 2006, Réseaux techniques et conflits de pouvoir: les dynamiques historiques des villes à l'époque contemporaine, Rome, Ecole française de Rome.

Bullock N, 1999, "A short history of everyday Berlin », In Goodman D. et Chant C. (sous la direction de), European Cities and Technology, Londres, Routledge.

CALABI D., 1980, "I servizi tecnici a rete e la questione della 
municipalizzazione nelle città italiane (1880-1910) », In Le macchine imperfette. Architettura, programma, istituzioni nel XIXe secolo, sous la direction de Morachiello P. et Teyssot G., Rome, Officina, pp. 293-332.

Caron F., Dérens J., Passion L., Cebron de Lisle Ph. (sous la direction de), 1990, Paris et ses réseaux: naissance d'un mode de vie urbain. XIXe-XXe siècles, Paris, BHVP.

ChATZIS K., 2000, La pluie, le métro et l'ingénieur: contribution à I'histoire de I'assainissement et des transports urbains, Paris, L'Harmattan.

Clifton G., 1992, Professionalism, Patronage and Public Service in Victorian London. The Staff of the Metropolitan Board of Works 1856-1889, Londres, Athlnone.

COHEN W., 1998, Urban Government and the rise of the French City. Five Municipalities in the Nineteenth Century, NewYork, St Martin's.

Colombinı V. et Cutinı A., 1990, Lo sviluppo della città e le infrastrutture a rete, Rome, DEI.

CoutARD O. (sous la direction de), 1999, The Governance of Large Technical Systems, sous la direction d'O. Coutard, Londres, Routledge.

Coutard O. Hanley R. et Zimmerman R. (sous la direction de), 2005, Sustaining urban networks. The diffusion of large technical systems, Londres, Spon.

Cowan R. S., 1997, A social history of American Technology, Oxford, Oxford University Press.

Dagenais M., Maver I. et Saunier P.Y. (sous la direction de), 2003, Municipal Services and Employees in the Modern City. New Historic Approaches, Londres, Ashgate.

Dogliani P. et Gaspari O. (sous la direction de), 2003, L'Europa dei Comuni dalla fine dell'Ottocento al secondo dopoguerra, Rome, Donzelli.

Dupuy G., 1988, Réseaux territoriaux, Caen, Paradigme.

Dupuy G., 1987, "Les réseaux techniques sont-ils des réseaux territoriaux? ", L’Espace Géographique, XVI, n³.

Dupuy G., 1991, L'urbanisme des réseaux: théories et méthodes, Paris, A. Colin

Dupuy G. et Knaebel G., 1982, Assainir la ville hier et aujourd'hui, Paris, Dunod.

Ethington P., 1994, The Public City. The Political Construction of Urban Life in San Francisco (1850-1900), Berkeley, California UP.

Fernandez A., 2006, "Compagnies privées et municipalités. Enjeux de pouvoir autour de l'installation des réseaux techniques dans les villes espagnoles », In Bocquet et Fettah.

FisCher C., 1982 : To Dwell Among Friends: Personal Networks in Town and City, Chicago, University of Chicago Press.

FISCHER C., 1994, America Calling: Social History of the Telephone to 1940, Berkeley, University of California Press.

Gaspari O., 2002, "Cities against States? Hopes, Dreams and
Shortcomings of the European Municipal Movement, 19001960 », Contemporary European History, 11-4, pp. 597621.

GheraRDI R., 1993, L'arte del compromesso. La politica della mediazione nell'Italia liberale, Bologne, II Mulino.

Graham S. et Marvin S., 2001, Splintering urbanism. Networked infrastructures, technological mobilities and the urban condition, Londres, Routledge.

Guerrand R. H., 1992, Moeurs citadines, Paris, Quai Voltaire.

Guillerme A., 1983, Les temps de l'eau. La cité, l'eau et les techniques, Seyssel, Champ Vallon.

Guy S. MARVIN S. et MOSS T., 2001, Urban Infrastructure in Transition, Londres, Earthscan.

HARTER H., 2001, Les ingénieurs des travaux publics et la transformation des métropoles américaines, Paris, Sorbonne.

HAYS S., 1998, Explorations in Environmental History, Pittsburgh, University of Pittsburgh Press.

Heynen N., Kaika M., et Swyngedow E. (sous la direction de), 2006, In the nature of cities. Urban political ecology and the politics of urban metabolism, Londres, Routledge.

Hugues T.P. (sous la direction de), 1964, The development of Western technology since 1500, New-York, Macmillan.

Hughes T. P., 1983, Networks of Power: electrification in Western society, 1880-1930, Baltimore, Johns Hopkins.

HuGhes T. P., 1990, Ein Mann vieler Eigenschaften: Walther Rathenau und die Kultur der Moderne, Berlin, Wagenbach.

Hughes T. P., 2004, American Genesis: a century of invention and technological enthusiasm, 1870-1970, Chicago, University of Chicago Press

Hughes T. P., 2004, Human-built world: how to think about technology and culture, Chicago, University of Chicago Press.

Hughes T. P. et Hughes A. C., 1990, Lewis Mumford: public intellectual, Oxford, Oxford University Press.

Hughes T. P. et MAYNTz R. (sous la direction de), 1989, The development of large technical systems, Boulder, Westview.

INGOLD A., 2003, Négocier la ville. Projet urbain, société et fascisme à Milan, Rome, Ecole française de Rome.

JACOBSON C. D., 1995, Ownership and financing of infrastructures: historical perspectives, Washington, World Bank.

Jacobson C. D., 2000, Ties that Bind. Economic and Political Dilemmas of Urban Utility Networks (1800-1990), Pittsburgh, University of Pittsburgh Press.

JACQUEMET G., 1979, «Urbanisme parisien: la bataille du toutà-l'égout à la fin du XIXe siècle », R.H.M.C., XXVI, OctDéc., pp. 505-548.

JaCQuot A., 2002, "La Compagnie Générale des Eaux. 18521952 : un siècle des débuts à la renaissance », Entreprises et Histoire, n³0, pp. 32-44.

JOERGES B., 1999, "High variability discourse in the history and 
sociology of large technical systems », In Coutard, 1999, pp. 258-290.

KAIKA M. et SWYNGEDOW E., 2000, "Fetishizing the Modern City: The Phantasmagoria of Urban Technological Networks », IJURR, 24-1; pp. 121-138.

KüHL U., 2001, Munizipalsozialismus in Europa, Munich, Oldenbourg.

Laroulandie F., 1993, "Les égouts de Paris au XIXe siècle. L'enfer vaincu et I'utopie dépassée », Cahiers de Fontenay, n69-70, mars, pp. 107-140.

LEMIRE V., 2006, " Histoire des réseaux techniques dans la municipalité ottomane de Jérusalem: Enjeux de souveraineté, conflits de pouvoirs, réseaux de mémoires », In Bocquet et Fettah, 2006.

LORRAIN D., 1997, "Le politique à tous les étages », In Villes en Europe, sous la direction de Bagnasco A. et Le Galès P., Paris, Découverte, pp. 201-229.

LORRAIN D., 2002, "Capitalismes urbains : la montée des firmes d'infrastructures », Entreprises et Histoire, n³0, p. 7-31.

LORRAIN D., 2005, " La firme locale-globale: Lyonnaise des Eaux (1980-2004) », Sociologie du Travail, 47, pp. 340-361.

LuCCARELl M., 1995, Lewis Mumford and the Ecological Region: the Politics of Planning, New-York, Guilford.

LuCkin B., Massard-Guilbaud G., SChOtT D., 2005, Resources of the city: contributions to an environmental history of modern Europe, Aldershot, Burlington.

MAlatesta M. (sous la direction de), 1995, Society and the Professions in Italy, 1860-1914, Cambridge, CUP.

MARIÉ et GARIÉPY M., 1997, Ces réseaux qui nous gouvernent?, Paris, L'Harmattan.

MATHEU M., 2002, "La régulation des services publics en réseau, ou la lente émergence d'une innovation majeure", Entreprises et Histoire, n³0, pp. 115-135.

MAYNTZ R., 1978, Vollzugprobleme der Umweltpolitik, Stuttgart.

MAYNTZ R., 1981, Kommunale Wirtschaftsförderung, Stuttgart, Kohlhammer.

MelIS G., 1999, Etica pubblica e amministrazione. Per una storia della corruzione nell'Italia contemporanea, Naples, CUEN.

Melosı M., 2000, The Sanitary City: Urban Infrastructure in America from Colonial Times to the Present, Baltimore, John Hopkins.

Melosı M., 2001, Effluent America. Cities, Industry, Energy and the Environment, Pittsburgh, University of Pittsburgh Press.

Melosı M., Scarpino Ph, (sous la direction de), 2004, Public History and the Environment, Malabar (Flo.), Krieger.

Millward R., 2000, "The political economy of urban utilities », In The Cambridge Urban History of Britain, sous la direction de Clark P., vol III dirigé par Daunton M., Cambridge, CUP.
Meriggi M. et Schiera P., 1993 (sous la direction de), Dalla città alla nazione. Borghesie ottocentesche in Italia $\mathrm{e}$ in Germania, Bologne, Il Mulino.

Neri Serneri S., 2002, Storia dell'ambiente e storia del territorio. La Toscana contemporanea, Milan, Angeli.

NyE D., 1994, American technological sublime, Cambridge (Ma.), MIT; Nye D., 1990, Electrifying America. Social Meanings of a New Technology, Cambridge (Mass.), MIT

NyE D., 2003, America as a second creation: technology and narratives of new beginnings, Cambridge (Ma.), MIT.

Offner J.-M. et Pumain D. (sous la direction de), 1996, Réseaux et territoires. Significations croisées, La Tour d'Aigues, L'Aube.

Pacey A., 1983, The Culture of Technology, Cambridge (Mass.), MIT.

Petitet S., 2002, "Problèmes et limites de la diffusion internationale d'un modèle de gestion des services publics ", Entreprises et Histoire, n³1, pp. 25-37.

PICON A., 1992, L'invention de l'ingénieur moderne, Paris, ENPC.

PORTER D. H., 1998, The Thames embankment. Environment, Technology and Society in Victorian London, Akron (Ohio), The University of Akron Press.

ReID D., 1991, Paris Sewers and Sewermen. Realities and Representations, Cambridge (MA), Harvard University Press.

Rodgers D., 1998, Atlantic Crossings: Social Politics in a Progressive age, Cambridge (Ma.), Harvard University Press.

Romanelu R., 1989, Sulle carte interminate. Un ceto di impiegati tra pubblico e privato: i segretari comunali in Italia. 1860-1915, Bologne, II Mulino.

ROMANELLI R.,1995, II comando impossibile. Stato e società nell'Italia liberale, Bologne, II Mulino.

Rutherford J., 2004, The Tale of Two Global Cities: Comparing the Territorialities of Telecommunications Developments in Paris and London, Londres, Routledge.

SAUNIER P-Y (sous la direction de), 2002, Municipal Connections: Co-operation, Links and Transfers among European Cities in the Twentieth Century, Contemporary European History, Special Issue. Cambridge, Cambridge University Press, XI, 4.

SCherRer F., 1992, L'égout, patrimoine urbain. L'évolution dans la longue durée du réseau d'assainissement de Lyon, Thèse de doctorat d'urbanisme, Université de Paris-Val de Marne.

SChultz S., MC ShANe C., 1978, "To engineer the metropolis: sewers, sanitation and city planning in the late 19th century America », Journal of American History, 65, pp. 389-411.

SignORelli A., 1999, Tra ceto e censo. Studi sulle élites urbane nella Sicilia dell'Ottocento, Milan, F. Angeli.

SORESINA M. (sous la direction de), 1998, Colletti bianchi. 
Ricerche su impiegati, funzionari e tecnici in Italia fra '800 € '900, Milan, F. Angeli.

SORI E., 2001, La città e i rifiuti. Ecologia urbana dal Medioevo al primo Novecento, Bologne, II Mulino.

SuMmERTON J., 1992, District heating comes to town. The social shaping of an energy system, Linköping, Linköping University Press.

Summerton J. (sous la direction de), 1994, Changing Large Technical Systems, Boulder, Westview.

TARR J., 1971, A Study in boss politics: William Lorimer of Chicago, Urbana, University of Illinois Press.

TARR J., 1985, Infrastructure and urban growth in the nineteenth century, Chicago, Public Works Historical Society.

TARR J., 1996, The search for the ultimate sink: urban pollution in historical perspective, Akron, University of Akron Press.

TARR J. et Dupuy G. (sous la direction de), 1988, Technology and the rise of the networked city in Europe and America,
Philadelphie, Temple University Press.

Tetı V. (sous la direction de), 2003, Storia dell'acqua. Mondi materiali e universi simbolici, Rome, Donzelli.

Topalov C. (sous la direction de), 1999, Laboratoires du nouveau siècle. La nébuleuse réformatrice et ses réseaux en France (1880-1914), Paris, Ehess.

TYGIEL J., 1994, The Great Los Angeles Swindle, Oxford, OUP.

VARASCHIN D., 2002, " De la concession en Espagne et en Italie, XIXe-XXe siècles », Entreprises et Histoire, n³1, pp. 24-70.

VARNI A. et Melis G. (sous la direction de), 1999, Burocrazie non burocratiche. Il lavoro dei tecnici nelle amministrazioni tra Otto e Novecento, Florence, Rosenberg e Sellier.

VORMS C., 2005, "La pratica e la regola. Gestione e controllo pubblico dell'urbanizzazione non-pianificata nella periferia di Madrid ", Storia urbana, 108, pp. 39-51.

ZuCCONı G., 1999, La città contesa. Dagli ingegneri sanitari agli urbanisti (1855-1942), Milan, Jaca.

\section{NOTES}

(1) Sur la genèse et l'évolution de cette notion, voir aussi: Joerges B., 1999.

(2) Sur Mumford, voir aussi : Luccarelli, 1995.

(3) Voir aussi : Pacey, 1983 et Fischer, 1982 et 1994. Pour un exemple d'histoire sociale d'un réseau d'infrastructure: Summerton, 1992. Voir aussi : Cowan, 1997.

(4) Parmi les publications préparatoires à cette somme: Tarr J., 1985, Infrastructure and urban growth in the nineteenth century, Chicago, Public Works Historical Society. Voir aussi les actes du séminaire international "Les réseaux techniques urbains, histoire contemporaine ", tenu à Paris en décembre 1983 (Ecole Nationale des Ponts et Chaussées), avec des communications de Anthony Sutcliffe, Clay Mc Shane, André Guillerme, Jean-Pierre Goubert, Letty Anderson, Georges Knaebel, Joel Tarr, Martin Melosi, John Mc Kay, Alain Cottereau, Dominique Laroque, Catherine Bertho, Chantal de Gournay, Mark Rose, Harold Platt, Roselyne Messager et Gabriel Dupuy. Voir aussi: Ausubel et Herman, 1988.

(5) Par exemple: Dupuy, 1987, 1988, 1991. Voir aussi: Offner et Pumain, 1996 et Colombini et Cutini, 1990.

(6) Sur ces questions, voir aussi: Jacobson, 1995 ainsi que, du même, 2000. En France, voir les travaux de P. Griset, A. Beltran, D. Barjot et C. Bouneau par exemple.

(7) Voir aussi dans le même numéro: Coutard O. et Pflieger G., "Une analyse du rôle des usagers le développement des services en réseau en France ». De même: Jacquot, 2002, Matheu, 2002, Barjot, Petitet, Varaschin, 2002, Petitet, 2002. Pour le Canada: Armstrong et Nelles, 1986.

(8) Sur ce même sujet, voir aussi : Guerrand, 1992 et Reid, 1991. 